\title{
Behavioral changes in patients with diabetes during the COVID-19 pandemic
}

\author{
Miyako Kishimoto $^{1,2}\left(\mathbb{D} \cdot\right.$ Takuya Ishikawa $^{2} \cdot$ Masato Odawara $^{1,2}$
}

Received: 1 July 2020 / Accepted: 17 September 2020 / Published online: 30 September 2020

(C) The Japan Diabetes Society 2020

\begin{abstract}
Aims/Introduction Amid the coronavirus disease (COVID-19) pandemic, the Japanese government declared a state of emergency and urged people to stay at home to prevent disease transmission. Herein, we investigated this emergency situation's effect on diabetes patients' lifestyle and glycemic control

Materials and methods Diabetes patients who visited our hospital between April 1 and June 13, 2020, for a regular consultation were asked about changes in their physical activities and dietary habits during the state of emergency period.

Results Among 168 patients, 26 (15.5\%) gained $>2 \mathrm{~kg}$; HbA1c levels were elevated or decreased by $>0.2 \%$ compared to that at the last visit in 57 and 51 patients (Groups D and I), respectively. Group D patients were affected to a larger extent by changes in commuting (transition to teleworking) and closures of sport gyms than Group I patients. Increased snacks, sweets, total diet, and alcohol intake could have contributed to worsening of glucose control in Group D, whereas a healthy diet and less alcohol intake could have led to better glucose control in Group I.

Conclusion During the state of emergency period, decreased physical activity levels negatively affected glycemic control. However, despite changes in physical activity level, maintaining or improving dietary habits could lead to better glycemic control in diabetes patients. During this COVID-19 pandemic, more diabetes patients are likely to shift to teleworking and stay home for longer periods. Therefore, we should develop effective and feasible measures to promote exercise and dietary therapy, especially for those who engage in teleworking.
\end{abstract}

Keywords COVID-19 $\cdot$ Stay-at-home $\cdot$ Physical activity $\cdot$ Diet $\cdot$ Teleworking

\section{Introduction}

The coronavirus disease (COVID-19) is an infectious disease caused by a recently discovered coronavirus which was hitherto unknown before the outbreak began in Wuhan, China, in December 2019 [1]. The World Health Organization (WHO) declared COVID-19 to be a public health emergency on January 30, 2020, and subsequently graded it as a pandemic that affected many countries worldwide. As of September 9, 2020, there were 27,486,960 confirmed

Miyako Kishimoto

mkishimoto@iuhw.ac.jp

1 Clinical Research Center, Department of Medicine, International University of Health and Welfare, 8-10-16 Akasaka Minato, Tokyo 107-0052, Japan

2 Department of Internal Medicine, Sanno Hospital, 8-10-16 Akasaka Minato, Tokyo 107-0052, Japan cases of COVID-19, including 894,983 deaths that have been reported to the WHO [1].

In Japan, since early March, the government asked all school to close and encouraged companies to introduce remote-working systems to prevent COVID-19 spread. On April 7, the Prime Minister declared a state of emergency for Tokyo, Osaka, and five other prefectures, and since April 16 , the declaration was applied nationwide. On the basis of the declaration, citizens were instructed to stay at home and asked to refrain from nonessential outings. The Japanese government lifted the nationwide state of emergency on May 25, and the economy was gradually reopened. This unprecedented situation affected all Japanese people, including patients with diabetes, to various levels.

To understand how this declaration affects the lifestyle and glycemic control of patients with diabetes, we investigated changes in individual behavior, body weight, and glycosylated hemoglobin (HbA1c) levels of patients with 
diabetes during the period of the declaration of a state of emergency.

\section{Materials and methods}

\section{Subjects}

Study participants were patients with diabetes who visited the Sanno Hospital between April 1 and June 13, 2020 for a regular consultation and whose treatment of diabetes remained unchanged since February 2020. The individualized information of the patients, such as sex, age, occupation, height, body weight at the previous visit, body weight at the study visit, the body mass index (BMI) at the study visit, of the previous visit, that of the study visit, duration of diabetes, family history of diabetes, complications, and treatments was acquired from the chart records. Doctors in charge of the patients asked them the following questions during the outpatient clinic visit. (1) During the nationwide state of emergency and stay-at-home period, did you find any change in physical activity (PA)? (2) Is there anything that you have changed or started about PA to compensate for the negative impact of this period? (3) Did you find any change in dietary habit during this period? (4) Is there anything that you have changed or started about dietary habit to compensate for the negative impact of this period? (5) What is your comprehensive assessment of your PA and diet situation during this period? The answers for these questions were aggregated, and substantially similar answers were grouped and categorized.

\section{Statistical analyses}

The relationship between the changes in glycemic control (deterioration or improvement in HbA1c levels) and changes in PA levels or dietary habit were assessed via multiple logistic analysis with the use of SPSS Statistics version 27 (IBM, Armonk, NY, USA). A $P$ value of $<0.05$ was considered statistically significant.

The protocol for this research project was approved by a suitably constituted Ethics Committee of the Sanno Hospital (approval no. 20-S-5, Approval Date; June 29, 2020), and informed consent was obtained from all patients for study participation.

\section{Results}

In total, 168 patients (161, type 2 diabetes; 7 , type 1 diabetes) participated in this study. The characteristics of these patients are shown in Table 1. The HbA1c levels were elevated by more than $0.2 \%$ or decreased by more than $0.2 \%$ from that at their last visit in 57 and 51 patients (patients whose glycemic control deteriorated [Group D] and the group of the patients whose glycemic control improved [Group I]), respectively. The remaining 60 patients showed no remarkable changes in glycemic control (changes in $\mathrm{HbA} 1 \mathrm{c}$ levels were not more than $\pm 0.2 \%$ ) and are categorized as Group N. Among all participants, compared to their last visit, 26 patients $(15.5 \%)$ gained more than $2 \mathrm{~kg}$, whereas 12 patients (7.1\%) lost more than $2 \mathrm{~kg}$ during the period. Regarding individual groups, Group I lost $0.06 \pm 1.60 \mathrm{~kg}$; however, patients in Group D and Group N gained $0.84 \pm 1.60 \mathrm{~kg}$ and $0.33 \pm 1.40 \mathrm{~kg}$, respectively.

The representative answers for Questions 1, 3, and 5 are categorized in Tables 2, 3, 4, respectively. Regarding Question 2, the most cited answer (multiple answers were allowed) in all groups was "walking or jogging." Specifically, 19 patients (37.3\%) in Group I and 23 patients (38.3\%) in Group $\mathrm{N}$ went walking or jogging, whereas only ten patients (17.5\%) in Group D did so. The second most cited answer in all groups was "Muscle training such as push-ups, sit-ups, stretching, or squatting." Seven patients (13.7\%) in Group I, ten patients (16.7\%) in Group N, and eight patients (14.0\%) in Group D cited this answer. Regarding Question 4 , the most cited answer (multiple answers were allowed) in all groups was "I try to eat a lot of vegetables." Ten patients (19.6\%) in Group I and 11 patients (18.3\%) in Group N tried to increase vegetable intake, whereas only 5 patients $(8.8 \%)$ in Group D cited this answer. The second most cited answer in all groups was "I tried to reduce the total amount of the diet (especially carbohydrate intake)." Nine patients (17.6\%) in Group I, seven patients (11.7\%) in Group N, and only three patients (5.3\%) in Group D cited this answer.

For the multiple logistic regression analyses, we coded PA levels as $0=$ increased, $1=$ no change, and $2=$ decreased and diet habit as $0=$ improved, $1=$ no change, and $2=$ deteriorated. The results revealed that changes in PA level and dietary habits were significant determinants of group categorization (Group D or I) with the odds ratios of 5.03 (95\% CI: $2.20-11.54 ; \mathrm{P}<0.05)$ and 6.92 (95\% CI: 3.23-14.85; $\mathrm{P}<0.05)$, respectively.

\section{Discussion}

The COVID-19 pandemic has affected the global population including patients with diabetes. Behavioral management, including PA and dietary habits, is fundamental to achieving treatment goals for people with diabetes [2-7]. However, many patients with diabetes were unable to maintain their previous lifestyle during the nationwide state of emergency and stay-at-home period. Based on the results of our study, as shown in Table 2, the patients in Group D were affected more by the changes in commuting (transition to 
Table 1 Characteristics of the study participants

\begin{tabular}{|c|c|}
\hline Variables & Mean \pm SD or $N(\%)$ \\
\hline Age (years) & $62.1 \pm 12.3$ \\
\hline Sex (males/females) & $116 / 52$ \\
\hline Height (cm) & $166.5 \pm 8.08$ \\
\hline Body weight (kg) & $71.57 \pm 14.33$ \\
\hline $\operatorname{BMI}\left(\mathrm{kg} / \mathrm{m}^{2}\right)$ & $25.6 \pm 4.0$ \\
\hline HbA1c (\%) & $7.26 \pm 0.95$ \\
\hline Duration of diabetes (years) & $8.3 \pm 7.4$ \\
\hline Family history of diabetes $(-/+)$ & $62 / 106$ \\
\hline Diabetic retinopathy & $21(12.5)$ \\
\hline Diabetic nephropathy & $50(29.8)$ \\
\hline Diabetic neuropathy & $51(30.4)$ \\
\hline \multicolumn{2}{|l|}{ Treatment } \\
\hline Diet and exercise only & $23(13.7)$ \\
\hline DPP4-I & $79(47.0)$ \\
\hline Metformin & $88(52.4)$ \\
\hline Sulfonylurea & $21(12.5)$ \\
\hline SGLT2-I & $42(25.0)$ \\
\hline$\alpha-\mathrm{GI}$ & $9(5.4)$ \\
\hline Glinide & $4(2.4)$ \\
\hline Insulin & $23(13.7)$ \\
\hline GLP-1 RA & $11(6.5)$ \\
\hline \multicolumn{2}{|l|}{ Working style } \\
\hline Employed and still commuting as before & $32(19.0)$ \\
\hline Employed and now completely shifted to telework & $53(31.5)$ \\
\hline Employed and sometimes commuting and sometimes teleworking & $19(11.3)$ \\
\hline Self-employed & $8(4.8)$ \\
\hline Formerly employed, but now unemployed or absent from work & $16(9.5)$ \\
\hline Originally unemployed & $40(23.8)$ \\
\hline
\end{tabular}

Data are expressed as mean \pm standard deviation or number $(\%)$

$B M I$ body mass index, HbAlc glycosylated hemoglobin, DPP4-I dipeptidyl peptidase-4 inhibitor, $\alpha$-GI $\alpha$-glucosidase inhibitor, $S G L T 2-I$ sodium-glucose cotransporter-2 inhibitor, GLP-1 RA glucagon-like peptide-1 receptor agonist

Table 2 Representative answers to Question 1 "During the nationwide state of emergency and stay-at-home period, did you find any change in physical activity?

\begin{tabular}{|c|c|c|c|}
\hline Answers & Group D & Group N & Group I \\
\hline PA level in commutation was decreased due to a transition to teleworking & $29(50.9)$ & $24(40.0)$ & $18(35.3)$ \\
\hline $\begin{array}{l}\text { Already working at home or unemployed. Therefore, no change in commutation but } \\
\text { less PA due to stay-at-home }\end{array}$ & $8(14.0)$ & $10(16.7)$ & $8(15.7)$ \\
\hline $\begin{array}{l}\text { Already working at home or unemployed. Therefore, no change in commutation and } \\
\text { no change in PA level in daily life }\end{array}$ & $5(8.8)$ & $5(8.3)$ & $11(21.6)$ \\
\hline Commuting as usual. Therefore, no change in the PA level in commutation & $3(5.3)$ & $9(15.0)$ & $5(9.8)$ \\
\hline Working every day but less PA due to less work load & $3(5.3)$ & $5(8.3)$ & $1(2.0)$ \\
\hline PA level was decreased due to discontinuance of business & $2(3.5)$ & $6(10.0)$ & $6(11.8)$ \\
\hline PA level was decreased due to closure of sports gym or swimming pool & $14(24.6)$ & $11(18.3)$ & $2(3.9)$ \\
\hline PA level was decreased due to lack of leisure activities & $3(5.3)$ & $1(1.7)$ & $4(7.8)$ \\
\hline PA level was increased due to increased housework & $0(0.0)$ & $0(0.0)$ & $1(2.0)$ \\
\hline
\end{tabular}

The numbers in the right column indicate the numbers and percentages of the individual answers. Multiple answers were allowed

$P A$ physical activity 
Table 3 Representative answers to Question 3 "Did you find any change in dietary habits during this period?"

\begin{tabular}{|c|c|c|c|}
\hline Answers & Group D & Group N & Group I \\
\hline The amount of snacks or sweets increased & $28(49.1)$ & $11(18.3)$ & $3(5.9)$ \\
\hline The restaurants were closed and mostly I ate at home. Home-cooked meals were healthier & $5(8.8)$ & $17(28.3)$ & $16(31.4)$ \\
\hline No remarkable change in the portion or content of diet & $16(28.1)$ & $26(43.3)$ & $19(37.3)$ \\
\hline Meal times became irregular & $2(3.5)$ & $2(3.3)$ & $1(2.0)$ \\
\hline The total amount of diet was increased (including the cases where 2 meals changed to 3 meals per day) & $14(24.6)$ & $6(10.0)$ & $2(3.9)$ \\
\hline The total amount of carbohydrate intake was increased & $5(8.8)$ & $1(1.7)$ & $2(3.9)$ \\
\hline Frequency of using delivery food, takeout food, or lunch box at the convenience store was increased & $6(10.5)$ & $4(6.7)$ & $2(3.9)$ \\
\hline The amount of vegetable intake was decreased & $1(1.8)$ & $2(3.3)$ & $2(3.9)$ \\
\hline I lost appetite due to less PA or constipation. Therefore, the amount of food intake was decreased & $0(0)$ & $3(5.0)$ & $3(5.9)$ \\
\hline $\begin{array}{l}\text { Less opportunity to drink outside, but more at home. Therefore, the total amount of alcohol intake was } \\
\text { increased }\end{array}$ & $9(15.8)$ & $6(10.0)$ & $6(11.8)$ \\
\hline $\begin{array}{l}\text { Less opportunity to drink outside due to the cancelation of business trip or party. Therefore, the total amount of } \\
\text { alcohol intake was decreased }\end{array}$ & $9(15.8)$ & $13(21.7)$ & $13(25.5)$ \\
\hline
\end{tabular}

The numbers in the right column indicate the numbers and percentages of the individual answers. Multiple answers were allowed $P A$ physical activity

Table 4 Representative answers to Question 5 "What is your comprehensive assessment of your PA and diet situation during this period?"

\begin{tabular}{|c|c|c|c|}
\hline Answers & Group D & Group N & Group I \\
\hline $\begin{array}{l}\text { PA decreased, and the diet deterio- } \\
\text { rated }\end{array}$ & $29(50.9)$ & $9(15.0)$ & $3(5.9)$ \\
\hline $\begin{array}{l}\text { PA decreased, and no change in the } \\
\text { diet }\end{array}$ & $13(22.8)$ & $9(15.0)$ & $4(7.8)$ \\
\hline PA decreased, and the diet improved & $6(10.5)$ & $18(30.0)$ & $13(25.5)$ \\
\hline $\begin{array}{l}\text { No change in PA, and the diet } \\
\text { deteriorated }\end{array}$ & $4(7.0)$ & $1(1.7)$ & $0(0.0)$ \\
\hline No change in PA and the diet & $3(5.3)$ & $16(26.7)$ & $8(15.7)$ \\
\hline $\begin{array}{l}\text { No change in PA, and the diet } \\
\text { improved }\end{array}$ & $1(1.8)$ & $5(8.3)$ & 7 (13.7) \\
\hline $\begin{array}{l}\text { PA increased, and the diet deterio- } \\
\text { rated }\end{array}$ & $1(1.8)$ & $0(0.0)$ & $3(5.9)$ \\
\hline $\begin{array}{l}\text { PA increased, and no change in the } \\
\text { diet }\end{array}$ & $0(0.0)$ & $1(1.7)$ & $4(7.8)$ \\
\hline PA increased, and the diet improved & $0(0.0)$ & $1(1.7)$ & $9(17.6)$ \\
\hline
\end{tabular}

The numbers in the right column indicate the numbers and percentages of the individual answers

$P A$ physical activity

teleworking) and closures of sports gym than those in Group I. To compensate for the negative impact of this period in PA, more patients in Group I started walking or jogging than those in Group D. To identify the reasons that contributed to the worsening of glycemic control, we asked questions on the changes in dietary habits during the period. As shown in Table 3, increased amount of snacks, sweets, total diet, and alcohol intake could likely have contributed in worsening glycemic control in Group D, whereas a healthy diet at home and less alcohol intake seem to have led to better glycemic control in Group I. Compared to Group D, a greater proportion of the patients in Group I tried to eat sufficient amount of vegetables. The answers of self-estimation for Question 5, categorized in Table 4, indicates that nearly half of the patients in Group D assessed that their PA levels had decreased and that the quality of diet had deteriorated. In contrast, regardless of PA levels, patients who tried to maintain or even improve their diet were more likely to be included in Group I.

With regard to teleworking, a survey conducted by the Tokyo Metropolitan Government showed that the ratio of teleworking companies in the capital surged from $24.0 \%$ in March to $62.7 \%$ in April 2020 as the spread of COVID-19 expanded. Furthermore, many firms continue to push teleworking due to a reduced risk of infection, even after the state of emergency was called off. Therefore, more patients with diabetes are expected to shift to teleworking and are likely to stay home longer than they did before. To minimize the risk of reducing PA levels and aggravating dietary habits, we should educate individual patients by providing appropriate and feasible measures for exercise and dietary therapy during teleworking. There is plenty of useful information available to us, some of which comes from the International Diabetes Federation that recommends healthy nutrition tips and home-based exercise on their official website [8].

Our study had some limitations. First, the self-reported patients' comments about changes in PA and dietary habits were, subjective and not scientifically evaluated. Second, because this survey was carried out at a single medical institute, the sample size was small, and the participant selection was limited to the patients of our hospital who may have special characteristics, such as living or working in urban cities close to the hospital, in a developed area of Tokyo. Therefore, the results of the survey may not be generalized. 
In conclusion, we investigated changes in individual behavior and the $\mathrm{HbA} 1 \mathrm{c}$ levels of patients with diabetes during the state of emergency and found out that decreased PA levels negatively affected glycemic control. However, regardless of changes in the PA level, efforts to maintain or improve dietary habit can lead to better glycemic control. It is important to understand the behavioral changes of patients with diabetes in the real-world setting, especially during this unprecedented situation, to adequately support them to maintain appropriate glycemic control. Not only during the COVID-19 pandemic, but also in the future, more patients with diabetes are expected to shift to teleworking and likely to stay home for extended periods. Therefore, we should develop effective and feasible measures to promote exercise and dietary therapy, especially for this population who engage more than ever in teleworking.

Acknowledgements The authors would like to thank all the subjects who participated in the present study.

Author contributions All authors contributed to the study conception and design. Material preparation and data collection were performed by MK. Statistical analysis was performed by TI. The first draft of the manuscript was written by MK and all authors commented on previous versions of the manuscript. All authors have read and approved the final manuscript.

\section{Compliance with ethical standards}

Conflict of interest All authors declare no conflict of interest.

Human rights statement All procedures followed were in accordance with the ethical standards of the responsible committee on human experimentation (institutional and national) and/or with the Declaration of Helsinki of 1964 and its later amendments.
Informed consent Informed consent or substitutes for it was obtained from all patients for the study participation.

\section{References}

1. World Health Organization. Coronavirus disease 2019 (COVID19) Situation Report. 2020.

2. Araki E, Goto A, Kondo T, et al. Japanese clinical practice guideline for diabetes 2019. Diabetol Int. 2020;11:165-223.

3. American Diabetes Association. Lifestyle Management: Standards of Medical Care in Diabetes-2019. Diabetes Care. 2019;42(Suppl. 1):S46-60.

4. American Diabetes Association. 2. Classification and Diagnosis of Diabetes: Standards of Medical Care in Diabetes-2020. Diabetes Care. 2020; 43(Suppl 1):S14-31.

5. Franz MJ, MacLeod J, Evert A, et al. Academy of Nutrition and Dietetics Nutrition Practice Guideline for Type 1 and Type 2 Diabetes in adults: systematic review of evidence for medical nutrition therapy effectiveness and recommendations for integration into the nutrition care process. J Acad Nutr Diet. 2017;117:1659-79.

6. Umpierre D, Ribeiro PAB, Kramer CK, et al. Physical activity advice only or structured exercise training and association with hba1c levels in type 2 diabetes: a systematic review and metaanalysis. JAMA. 2011;305:1790-9.

7. Johansen MY, MacDonald CS, Hansen KB, et al. Effect of an intensive lifestyle intervention on glycemic control in patients with type 2 diabetes: a randomized clinical trial. JAMA. 2017;318:637-46.

8. International Diabetes Federation: home-based exercise for people with diabetes. https://www.idf.org/aboutdiabetes/what-is-diabetes/ covid-19-and-diabetes/home-based-exercise.html

Publisher's Note Springer Nature remains neutral with regard to jurisdictional claims in published maps and institutional affiliations. 